\title{
Stag beetle elytra: localized shape-retaining, puncture and wear-resistant
}

\author{
Lakshminath Kundanati ${ }^{1}$ Roberto Guarino ${ }^{1}$ and Nicola M. Pugno ${ }^{1,2,3^{*}}$ \\ 1 Laboratory of Bio- Inspired \& Graphene Nanomechanics, Department of Civil, Environmental and \\ Mechanical Engineering,University of Trento, via Mesiano, 77, 38123, Italy; 1.kundanati@unitn.it (LK), \\ roberto.guarino@unitn.it (RG). \\ 2 School of Engineering and Materials Science, Queen Mary University of London, Mile End Road, London \\ E1 4NS, United Kingdom; \\ 3 Ket Lab, Edoardo Amaldi Foundation, Via del Politecnico snc, 00133 Rome, Italy \\ * Correspondence: nicola.pugno@unitn.it
}

\begin{abstract}
Beetles are by far one of the most successful and diverse insect species. A part of this success is attributed to their elytra which provide various functions such as protection to their bodies from mechanical forces and the harmful environmental factors. In this study, Stag beetle (Lucanus cervus) elytra were first examined for their overall flexural properties and were observed to have a localized shape retaining snap-through mechanism which could play a crucial role in energy absorption, e.g. during battles and falls from heights. The snap-through mechanism was validated using theoretical calculations and also finite element simulations. Elytra were also characterized to examine their puncture and wear resistance. Our results show that elytra resisted puncture up to a force of $1.8 \pm 0.4 \mathrm{~N}$ and have puncture resistance compared to that of commercially available puncture resistant gloves. The measured values of modulus and hardness of elytra exocuticle were $10.3 \pm 0.8 \mathrm{GPa}$ and $0.7 \pm 0.1 \mathrm{GPa}$. Using the hardness to modulus ratio as an indicator of wear resistance, the estimated value was observed to be in the range of wear resistant biological materials. Thus, our study demonstrates different mechanical properties of the stag beetle elytra which can be explored to design shape retaining bio-inspired composites with enhanced puncture and wear resistance.
\end{abstract}

Keywords: Beetle elytra; snap-through; puncture-resistant 


\section{Introduction}

Insects are the most successful group of organisms on Earth accounting to almost $90 \%$ of all species and are also highly diverse organisms. Their survival can be attributed to their small body, exoskeleton, ability to fly and adapt to the changing environment. Of them, beetles are the most diverse group of species of insects. This extraordinary diversity is attributed to their highly sclerotized strong fore wings, which protect the wings when they are not is use and to their powerful flight using their membranous hind wings [1]. The forewings termed as "elytra" play an important role in the survival of beetles especially in battles and protection from the harmful environment outside. Elytra are multi-layered composites made of chitin fibrils and protein matrix [2] and were examined for their microstructure and mechanical properties [3-5]. Therefore, elytra are biological composites of importance for bio-inspired designs and it is crucial to perform a comprehensive mechanical characterization from a functional perspective.

In engineering structures, instabilities such as buckling and snap-through are usually considered as a disadvantage but recently novel ideas are presented for the use of such mechanisms. For instance, buckling phenomenon is exploited in the design of energy dissipation mechanisms such as absorbers, isolators, and stabilizers [6] and snap-through instability is used for amplifying the response of a fluid-based actuator [7]. A bi-stable mechanism is observed in the beams that present two stable states, such as positions A and D (Figure 1A). In between, two intermediary states are observed in which snap through and reverse snap-through mechanisms are activated during deformation, referring to positions $B$ and $C$, respectively. In displacement control conditions, the loaded beam traverses the path A-B-C-D (blue arrows). On the contrary, in force control conditions, the snapthrough behavior is displayed by the path A-B-D (green arrows). This behavior allows the beam to absorb the released kinetic energy (Figure 1B). In this phenomenon, shape change occurs either in unstable or stable manner and either reversibly or irreversibly. In such morphing structures, the shape change occurs either actively or passively depending on various factors such as intrinsic material behavior, geometry, temperature, load, and possibly some kind of actuation that enables switching between the two states [8]. Using inspiration from such structures, a pseudo bi-stable dome-shaped structure is developed that can remain in the deformed state and recover to its original shape without any actuation [8]. It is also possible to have more than one stable state of deformation in non-symmetric fiber reinforced composites because of the residual stress developed due to thermal, moisture and chemical effects [9]. In such composites, switching from one stable state to the other is possible through a snap-through mechanism with the aid of a force. Such composites materials can be used to develop force-mediated shape-adaptive materials.

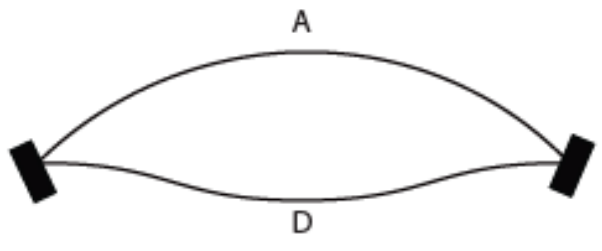

A.

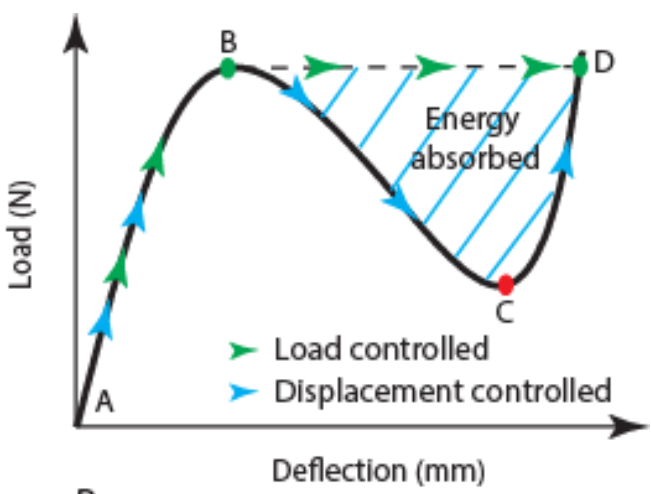

B.

Figure 1. A) Schematic of a shallow structure in the initial and final configuration. B) The corresponding response is shown in the load-deflection curve.

Energy absorption designs that are aimed at preventing catastrophic failures use either microscopic or macroscopic deformation mechanisms. In biological materials such as bone, it is driven by the deformation at the micro-scale, such as delamination and micro-buckling of lamellar 
structures that are controlled by the distribution of tubules [10]. Recently, energy absorption capabilities are achieved by macroscopic deformation of honeycomb structures with negative stiffness, fabricated using pre-curved beams [11]. Using such ideas, a negative stiffness honeycomb structure is developed that can absorb a large amount of energy at a stable stress value, capable of recovering from large deformations. In Nature, thin sheets with curvature are common [12] but very few of them deform using snap-through mechanisms. One such example includes the fast leaf closure of Venus flytrap plant in an active way [13].

Protective armors are found across in many animals such as armadillo and turtles [14,15]. This is achieved by having high wear and puncture resistance of the skin. The wear response of a biological material is dependent on the mechanical function it is used for, such as grasping, biting or protecting soft body [16]. For example, the components of the head articulation system of a beetle were observed to be in permanent contact and therefore should be resistant to wear [17]. Elytra were shown to play an important function as a protective cuticle for the delicate wings and the abdomen of the Japanese rhinoceros (Allomyrina dichotoma) beetle [18]. There are a few studies aimed at studying puncture resistant mechanisms in fish scales and cockroach abdomen cuticle [19,20].

Earlier studies measured the mechanical properties of elytra but very few have tested the mechanical response of its whole structure. In this study, we demonstrated the snap-through and reverse snap-through mechanisms of the stag beetle elytra, which enable energy absorption during impacts and yet retain shape. Finite element simulations and analytical modelling of the snapthrough phenomenon were performed to validate the experimental results. We also performed experiments to test its protective capabilities using puncture tests and hardness measurements. To the best of our knowledge, this is the first time that such a snap-through mechanism is highlighted in insect cuticle. We hope this study not only contributes to the existing knowledge but also to the development of bio-inspired shape-retaining composites with protective capabilities.

\section{Materials and Methods}

\subsection{Sample preparation}

Male stag beetle samples were obtained in dehydrated state from the collection of the MUSE Science Museum of Trento (Trento, Italy). Rectangular sections were cut out of the elytra separated from the insect (Figure 2A). The schematic of the insect abdomen cross-section region shows the stack up of the wing and elytra on the abdomen surface (Figure 2B). The sectioned samples were clamped to restrict the in-plane movement of the ends and actuation is done by using an acrylic blade to create a line contact on the external surface (Figure 2C). The acrylic blade was held by metal clamps that were connected to the load cell. In the whole elytra experiments, the samples were directly used after separating them from the insect body.

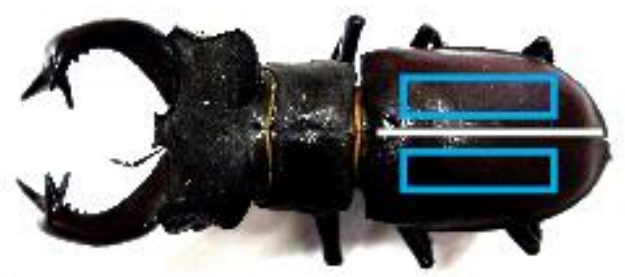

A.

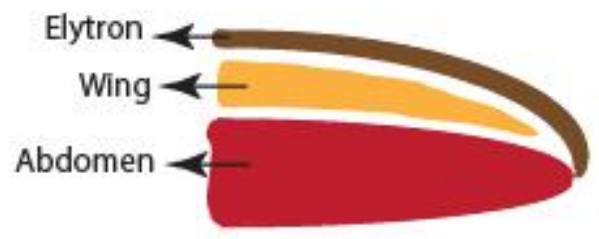

B.
C.

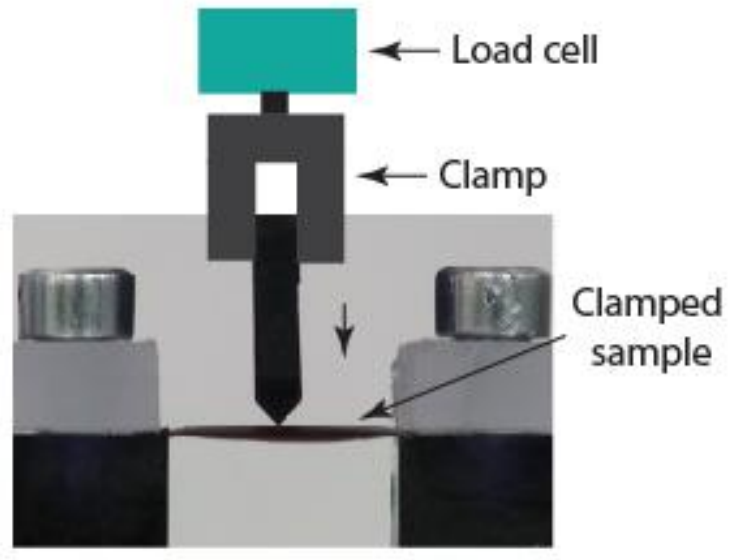


Figure 2. A) Sample extraction location for the experiments (blue boxes). B) Schematic of crosssectional view of the insect body along the axis of symmetry of the body (white line in A). C) Clamp set-up used for performing the experiments.

\subsection{Optical and Electron microscopy}

The images of the insect and the microstructure of the elytra were captured using a Lynx LM-1322 optical microscope (Olympus corporation, Japan) and a CCD camera (Nikon. Japan) attached to the microscope. Scanning Electron Microscope (SEM) imaging of all the samples is performed after cleaning with the help of ultrasonication and a subsequent drying. The prepared elytra sections were carefully mounted on double-sided carbon tape, stuck on an aluminum stub followed by sputter coating (Manual sputter coater, Agar Scientific Ltd, United Kingdom) with gold. Images were captured using an SEM (EVO 40 XVP, ZEISS, Germany) with accelerating voltages between 5 and 20 $\mathrm{kV}$. Image software was used for all dimensional quantification reported in this study (Abràmoff and Magalhães, 2004).

\subsection{Mechanical testing}

All the mechanical tests were performed using a Messphysik MIDI 10 (MESSPHYSIK, Materials Testing GmbH, Germany) Universal Testing Machine and the forces were obtained using a $\pm 10 \mathrm{~N}$ transducer (Leane International Srl, Italy). A qualitative approach is used to examine the whole elytra deformation by subjecting them to point loading. The objective is to try to simulate the loading from e.g. the opponent male beetle mandible or impact loading during the fall from heights during battles on the trees. So, we performed point-force compression tests on the whole elytra ( 2 tests per single elytron) by placing it on a soft polymer substrate to mimic the softer abdominal structure, at a rate of $0.01 \mathrm{~mm} / \mathrm{sec}$. Bending experiments were performed on the mechanical cut samples ( $\mathrm{N}=2$ samples, 2 tests each) to determine the snap-through behavior. These tests were performed on a custom-built bending set up machined out of hard plastic material with the possibility to clamp the sample edges. The rate of testing in 3-point bending tests was kept at $0.01 \mathrm{~mm} / \mathrm{s}$. The puncture tests $(\mathrm{N}=10$, with 2 needles and 5 samples cut from each beetle) were performed using a $21 \mathrm{G}$ gauge needle that was connected to the same transducer (LEANE Corp., $\pm 10 \mathrm{~N})$ and the displacement $(0.005 \mathrm{~mm} / \mathrm{s})$ is controlled using the same Messphysik MIDI 10 Universal Testing Machine.

\subsection{Finite element simulation}

Elytra have a complex geometry and anisotropic material properties that are similar to those of composite laminates. We simplified the model for the finite element numerical simulations by considering the geometry as a simple curved plate, with average thickness $t=0.27 \mathrm{~mm}$ (also used the variation in thickness along the length), half-length $1=4.6 \mathrm{~mm}$, half-width $\mathrm{w}=1.98 \mathrm{~mm}$ and maximum out-of-plane height $\mathrm{h}=0.43 \mathrm{~mm}$ (with respect to the bottom surface), taken from the experimental setup. We assigned isotropic material properties, with Young's modulus $\mathrm{E}=650 \mathrm{MPa}$ (i.e. around the average of the measured flexural moduli [21]). The values of Poisson's ratio $(v=0.1)$ and density $(\varrho=$ $1425 \mathrm{~kg} / \mathrm{m} 3$ ) chosen to be in the order of chitin properties [22], as a first approximation. We used a simplified symmetric model representing one quarter of the complete geometry and specified two symmetry planes (Figure 3). Identical to the experimental setup, the model was clamped at both the ends and the load was applied in displacement-control mode over a transversal line at the middle of the top surface. The total load was obtained following an incremental approach known as the Riks method [23]. The computational volume was discretized with 9000 second-order tetrahedral elements. 


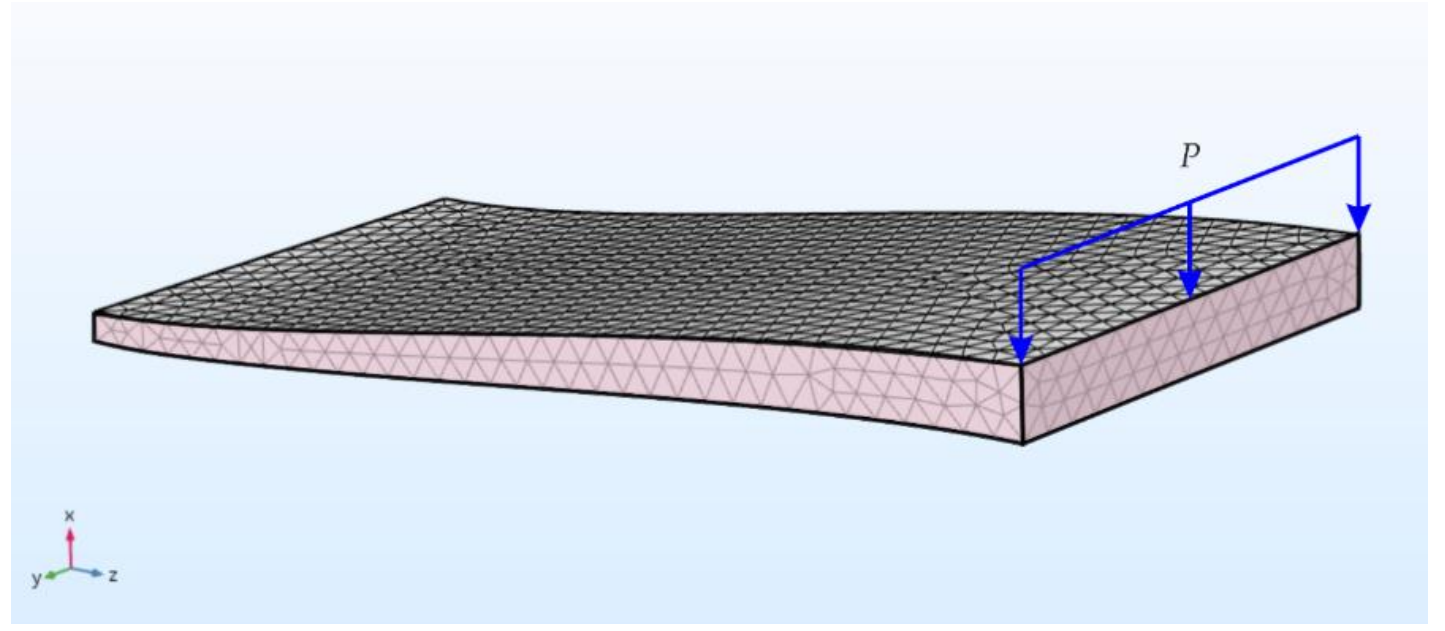

Figure 3. Meshed model and coordinate reference system, with highlighted the two symmetry planes (pink) and the applied edge load P.

\subsection{Nanoindentation}

The dried elytra were sectioned in the thickness direction after embedding them in resin and later were polished using a series of 400, 800, 1200, 2000 and 4000 grade sand papers. Finally, the samples were polished using a diamond paste of particle sizes in the range of $6 \mu \mathrm{m}$ and $1 \mu \mathrm{m}$, to obtain a surface of minimal roughness. The exocuticle region was carefully selected using optical microscope and the indentations were performed at this location. A Berkovich indenter was used to perform nanoindentation experiments employing a maximum load of $20 \mathrm{mN}$ at the rate of $1200 \mathrm{mN} / \mathrm{min}$. We performed a total of 12 indentations (with 4 indentations at each of the 3 different locations).

\section{Results}

3.1 Microstructure of the elytra and the abdominal surface

We examined the structural features on the abdomen and the elytra that help in keeping them locked together, when the beetle is not in flight (Figure 4A). Our results show that elytra comprise primarily three different bulk layers. A top layer, which is exposed to the environment, followed by a middle layer and a relatively thin layer that is connected to the middle layer by trabecular structures (Figure $4 \mathrm{~B})$. These agree with the observations made in other beetle elytra [3]. Elytra and abdomen have primarily four contact regions that help in mitigating the forces. The first contact is the direct physical connection of the elytra to the thorax ( $\mathrm{C} 1$, Figure $4 \mathrm{~A})$. The second contact is the locking mechanism between the left and right elytron that runs along the inside (C3, Figure $4 \mathrm{~A})$. These locking mechanisms were studied earlier and they require certain amount of force to decouple the elytra [24]. The third contact is a triangular shaped structure which protrudes from the backside of the head, under which the top inner-side corners of the elytra get tucked in (Figure 4C-D). The fourth contact region is the abdomen edge surface (Figure $4 \mathrm{C}$ ) regions with microtrachia that assist in friction-based locking (Figure 4E). Such microtrachia were also reported in the tenebrionid beetles [25]. Though some of these features were reported earlier in other beetle systems, we report them again here from Lucanus cervanus and also the other microstructures that possibly work together in keeping the elytra in contact with the abdomen firmly. 


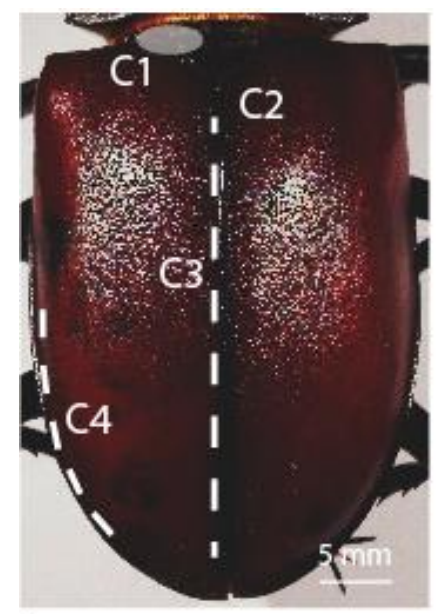

A.

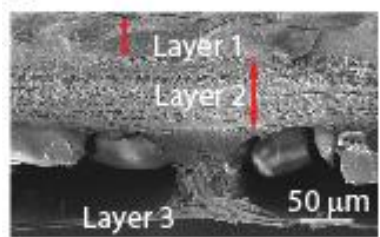

B.

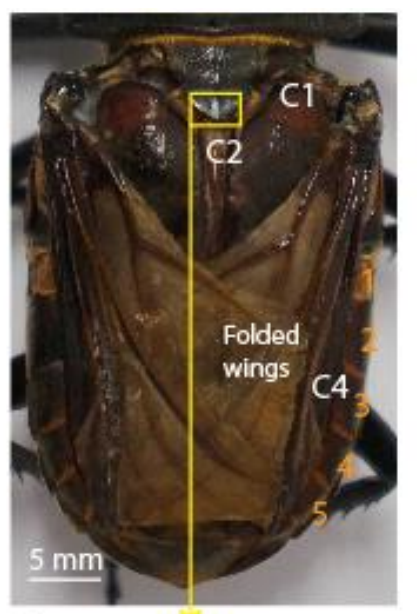

C.

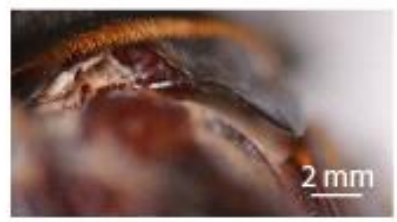

D.
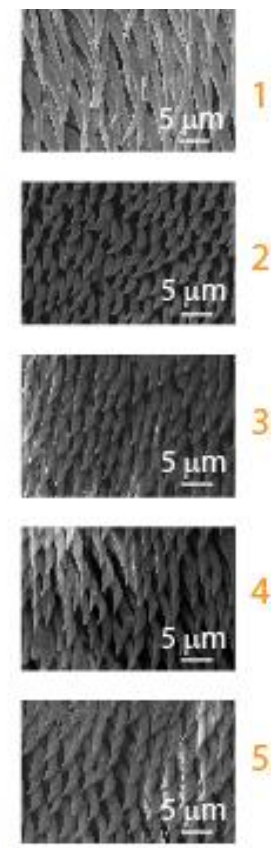

E.

Figure 4. A) Primary contact regions of elytra with abdomen. B) Scanning electron micrograph of the cross-section of an elytron highlighting the different layers. C) Abdomen surface with folded wings after removal of elytra showing the hinge location (C1), a triangular shaped structure (C2) which accommodates the inner-side corners of elytra and five surfaces with microtrachia (C4). D) Magnified image of C2. E) Scanning electron micrographs of the locking edge surfaces showing the microtrachia.

\subsection{Deformation of the elytra}

Loading experiments were carried to examine the overall mechanical behavior of the whole elytra. The force-deflection curves from these experiments showed that the elytra deform elastically with a snap-through and reverse snap-through behavior. The shape changes were reflected in loading curves (Figure 5A), with a snap-through occurring at point $\mathrm{B}$ and reverse snap-through occurring just before reaching point $C$. The shape changes of the elytra after the snap-through and reverse snapthrough were observed with a change in curvature (Figure 5B and 5C). It appears that the elytra work similar to the principle of bi-axial curvature, which is reported to be an efficient way of reinforcing thin sheet structures [12]. Attempts at fabricating morphing structures led to the development of a macro-fiber based two-layer cross-ply composite that morphs from one shape to another shape with the help of piezoelectric actuation [26]. On the contrary, the elytra appear to have a structural design that can switch back to the undeformed state without any active actuation system. The snap-through behavior in multiple ply composites is dependent on various factors like differences in Young's modulus along different directions, thickness of the ply and coefficients of thermal expansion [27]. The small defects in the material can also influence morphing in one or other stable states [28]. The response of the elytra resembled the mechanical response of a monostable buckling structure which has the capability to reverse snap-through after the load removal [29]. We showed the elytra can retain shape after absorbing the impact energy using a snap-through and reverse snap-through mechanisms even in an unconstrained condition as in our experiments. We can thus claim that the elytra when constrained on the beetle body with the microstructural features (Figure 4) it will offer higher resistance to deformation and there by absorb more energy when undergoes deformation. 


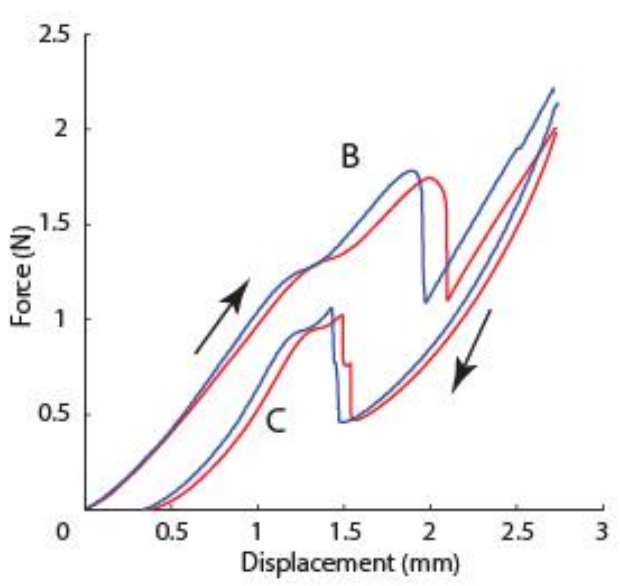

A. Loading and unloading response

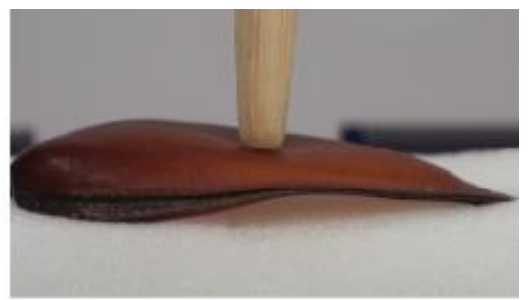

B. Buckled state

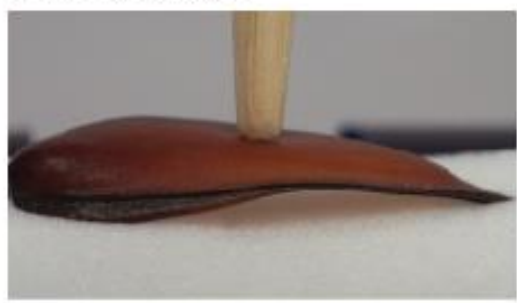

C. After recovery

Figure 5. Whole elytra deformation response during loading and unloading A) Force-displacement curves B) Elytron shape in the buckled state. C) Elytron shape recovery after reverse snap-through.

To perform mechanical analysis of the snap-through behavior of the elytra using a standard procedure, we performed the bending experiments by clamping the sectioned out rectangular samples at the ends. In clamped condition, the samples remained in the stable buckled state after removal of the load unlike the whole elytra samples. Force-displacement curves showed good repeatability in both the loading responses (Figure 6). The loading response in clamped condition showed that buckling occurs at a load of $0.37 \pm 0.02 \mathrm{~N}$ and is higher than the load in the buckled state.

An analytical analysis was performed to describe the experimental results in Figure 6A, based on the so-called Von Mises arch [30,31]. Elytra are characterized by a continuous geometry with structural imperfections and nonlinearities. Though the present shallow-arch model is an approximation, it has the capability to help in understanding the basic principles of snap-through instabilities. As shown in Figure 6 (inset), we approximate the elytron as two hinged bars loaded at the centre, with $\alpha$ the initial angle with respect to the horizontal plane. Under the assumption of small angles, the loaddisplacement relation is given by:

$$
F(\delta)=k_{a} \delta\left(2 \alpha^{2}-3 \alpha \frac{\delta}{l}+\frac{\delta^{2}}{l^{2}}\right)+2 k_{\theta} \frac{\delta}{l^{2}}
$$

where ka is the axial stiffness of the equivalent bar and $\boldsymbol{k}_{\boldsymbol{\theta}}$ is the rotational stiffness of the lateral hinges. Here we can assume $\boldsymbol{k}_{\boldsymbol{a}} \approx \boldsymbol{E} \boldsymbol{A} / \boldsymbol{l}=\boldsymbol{E} \mathbf{2} \boldsymbol{w} / \boldsymbol{l}$, where A is the cross section of the elytron and we have used the half-length 1 instead of the actual curve length because of the small initial angle. In general, an increasing rotational stiffness produces an increase in the critical load as well as in the critical displacement, thus all the curves tend to move towards the top right part of the diagram. The effect of the Young's modulus, instead, is simply to increase the critical load, without moving the position of the peak (i.e. the value of the critical displacement). Note that this reasoning is consistent with the buckling analysis of spherical shells, which can be considered an upper-bound estimate for our system and the details can be found elsewhere [32]. Also, in this case, the critical load is directly proportional to the Young's modulus of the shell structure, but the shape of the snap-through curves is heavily dependent on the geometry. Considering a Young's modulus of $1000 \mathrm{MPa}$, i.e. in the order of that measured value in a previous work [21], we can fit the average experimental curve with Equation (1), obtaining an axial stiffness $\mathrm{ka} \approx 175 \mathrm{~N} / \mathrm{mm}$ and a rotational stiffness $\mathrm{k} \theta \approx 5.2 \mathrm{Nmm} / \mathrm{rad}$ for the equivalent shallow-arch model, with R2-value 0.856. As shown in Figure 6, the model is able to match quite well the initial part of the experimental load-displacement curve, while a larger deviation from experiments is observed in the post-critical stage. On the contrary, the post-buckling 
behaviour can be captured sufficiently well by the finite-element simulations, with the simulated critical load in close agreement with the experimental data. Instead, the critical displacement value was found to be around half the corresponding experimental value.

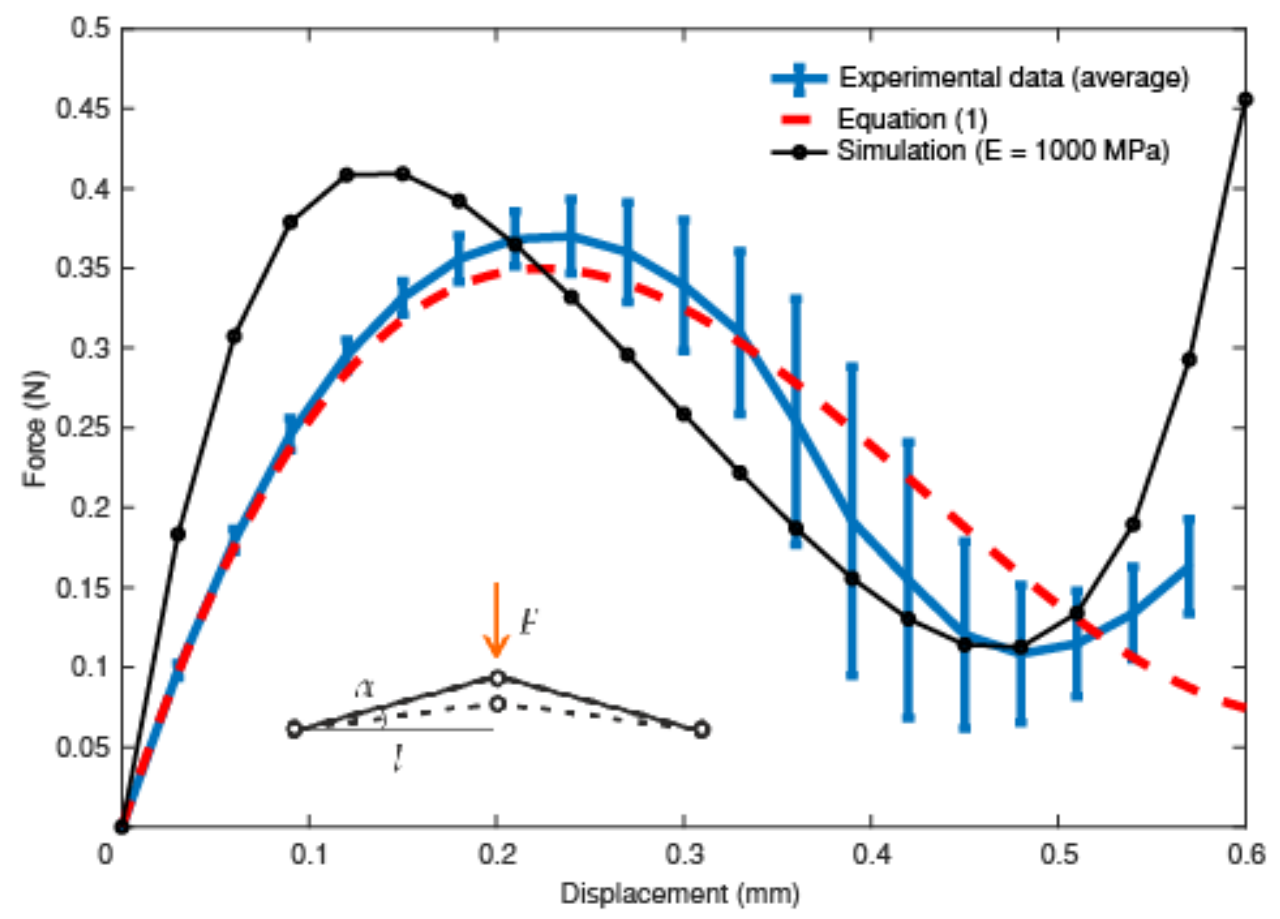

Figure 6. A) Comparison of force-displacement curves from the experiments, analytical estimation and the finite element simulation.

The observed differences between experiments, theory and simulations are due to the uncertainties in the geometry of the considered elytra and the presumably nonlinear material behavior, which do not allow us to make an extremely precise comparison. However, the simplified analytical model and the finite element simulations presented in this work still display a good potential in describing the observed experimental evidence. The variation is also attributed to assumption that the elytra is a homogeneous flat plate with uni-directional curvature as opposed to the bi-axial curvature and also their multilayered composite nature. This remains a limitation of the study but demonstrates the ability of simplified theoretical calculation to closely validate the experimental observation. Thus, elytra are structures with the ability to retain shape or recover from snap-through instantly unlike naturally existing venus-fly trap and the respective engineering composites. The multilayered structure of elytra appears to be similar to that of helmets that have a stiffer outer shell that enable impact distribution and thus avoiding concentrated loads [33]. Note that the experiments were carried out on naturally dried specimens because the selected species belongs to a near threated (IUCN Red list) state. Thus, the mechanical properties are expected to be higher than the cuticle in native state. The comparison of the experimental and analytical predictions remains still valid.

To understand the effect of dehydration of the cuticle, we performed additional simulations by changing the material properties such as the absolute value of the Young's modulus and orthotropocity. Our results showed that there is relevant change only in the slope of the first part of the curve and the absolute value of the critical load, without significantly affecting the critical displacement. Also, the introduction of orthotropic material properties, which are closer to the actual material, does not change the shape of the force-displacement curve (Figure 7). Although there is a significant change in critical values of load and displacement, the phenomenon of snap-through remains, showing that the elytra can still undergo this mechanism to absorb energy even in their natural state. 


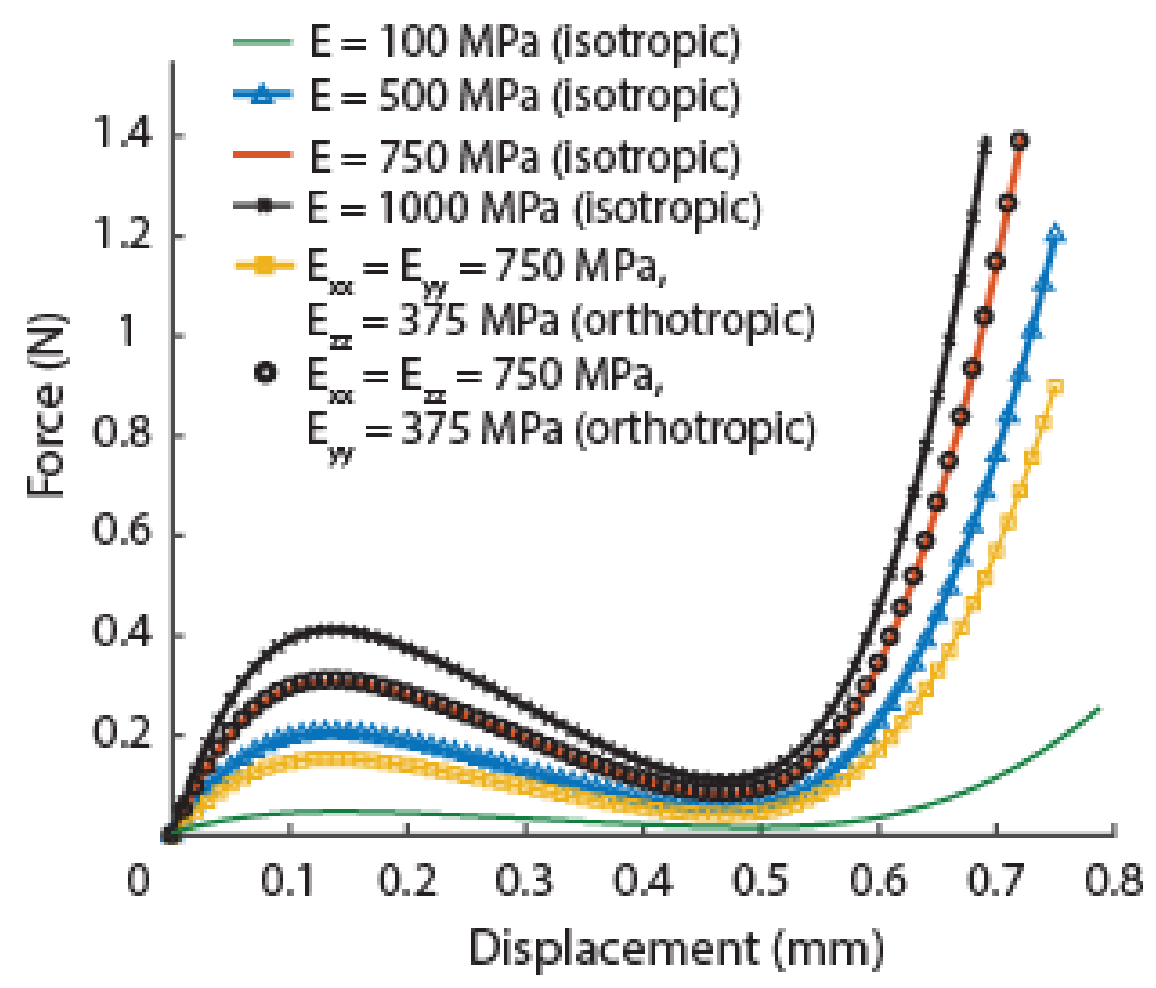

Figure 7. Force-displacement curves from finite element simulations for different material properties.

\subsection{Puncture and wear resistance}

Puncture experiments were performed on the elytra using a standard surgical needle (Figure 8A), as explained in Section 2. Results from the puncture experiments showed moderate repeatability (Figure $8 \mathrm{~B})$. The puncture force was defined at the initial deflection in the force-displacement curve which results in cracking of the relatively harder surface layer (Figure $8 \mathrm{~B}$, denoted by a star sign). The average values of the puncture force were observed to be $1.8 \pm 0.4 \mathrm{~N}$ (average over $\mathrm{N}=10$ tests). The variation in the force values can be attributed the regional variation in the microstructural features of the elytra, such as pore canals and trabecular structures. The penetration process is observed with the needle in contact with the top surface at the beginning and after the puncture (Figure 8C (i-ii)). The puncture force values of elytra are similar to that of artificial gloves (HexArmor 7084, 9014, www.hexarmor.com) designed to resist puncture with average puncture force of $2.6 \mathrm{~N}$ and $3 \mathrm{~N}$. The damage created from the puncture was shown in the image taken from the top surface of the sample, with brittle-like fracture of the top exocuticle layer (Figure 8D (i)). Similar brittle-like behavior of the top layer was also observed in a fractured sample surface (Figure 8D (ii)). Brittle behavior of the top layer (exocuticle) associated with the hardened nature of this layer is a consequence of cross-linking between helicoidally arranged nano-chitin fibrils and protein complex [34]. The primary resistance to puncture comes from the exocuticle, which also aids in improved wear resistance. Similar puncture tests were performed on three species of cockroach cuticle and puncture forces were measured to be between 1.2 to $30.7 \mathrm{~N}$ [20], though these cannot be directly compared due to the differences in needle characteristics.

We measured the indentation properties of the elytra external bulk layer to estimate the wear properties, using the embedded cross-section samples (Figure 9). The Young's modulus and hardness of this layer were found to be $10.3 \pm 0.8 \mathrm{GPa}$ and $0.7 \pm 0.1 \mathrm{GPa}$, respectively. These values were in the same range of hardest exocuticle layer (E 10 GPa, and H 0.4 GPa) of the Sun beetle (Pachnoda marginata) cuticle in the head articulation system [17] and close to the values (H 0.95 GPa) of crab claw in the exocuticle region [35]. Earlier studies report that the dehydrated cuticle hardness is an order of magnitude higher as compared to that of the natural state, specifically in endocuticle and 
meso-cuticle [36]. The effects were significantly lower in exocuticle with a factor of 2.4 in the values of $E$ and 1.6 in the hardness values. However, the measured hardness and Young's modulus values can be considered for the following estimation of wear and this variation is minimized because of the considered hardness to modulus ratio. The wear resistance can be roughly related with the ratio (H1.5/E) [37], which is calculated to be $0.05 \pm 0.01 \mathrm{GPa} 1 / 2$. These values were close to the values observed in Glycera jaw ( 0.077 GPa1/2) which is known to be wear resistant [38] and the high abrasion resistant (0.06-0.08 GPa1/2) outer layer of the spider fang [37]. Materials with design of a hard-external layer and relatively softer inner layers, enable them to be puncture resistant and also be flexible [39]. Thus, we can see from the above results that the elytra are designed to be both puncture and wear resistant.

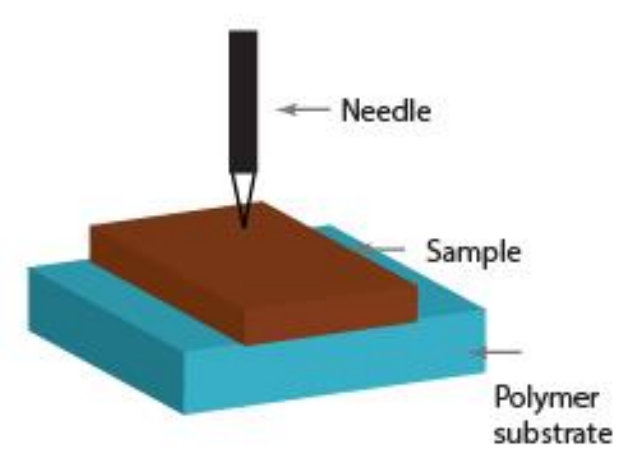

A. Puncturing experiment set up
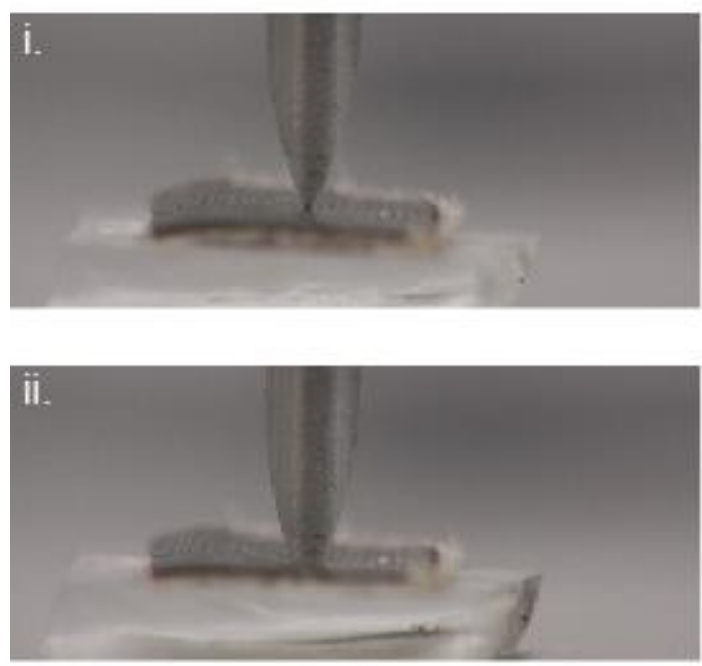

\section{Snap shots from the video i)before and ii) after punture}

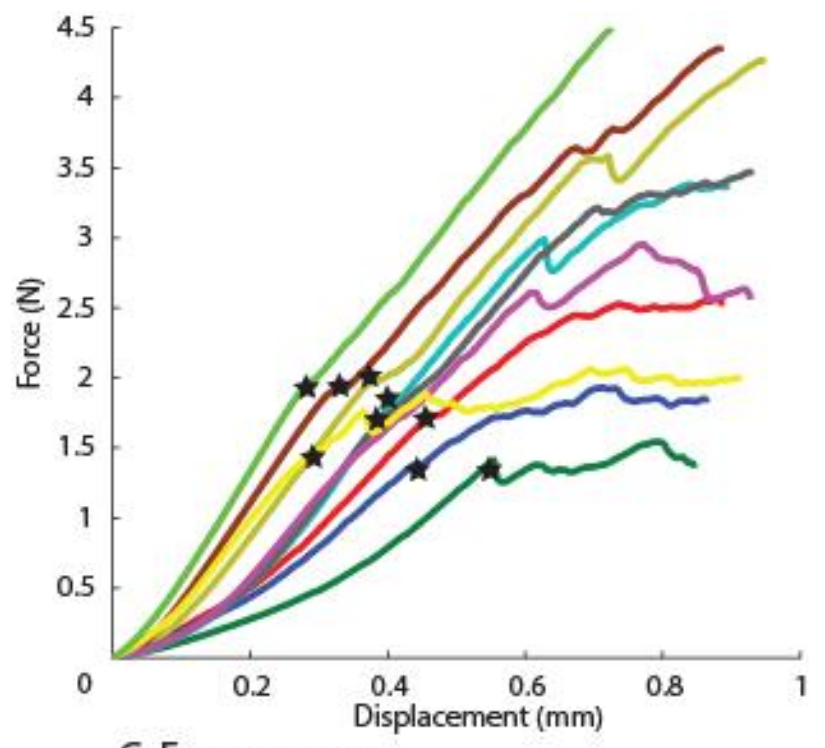

C. Force curves
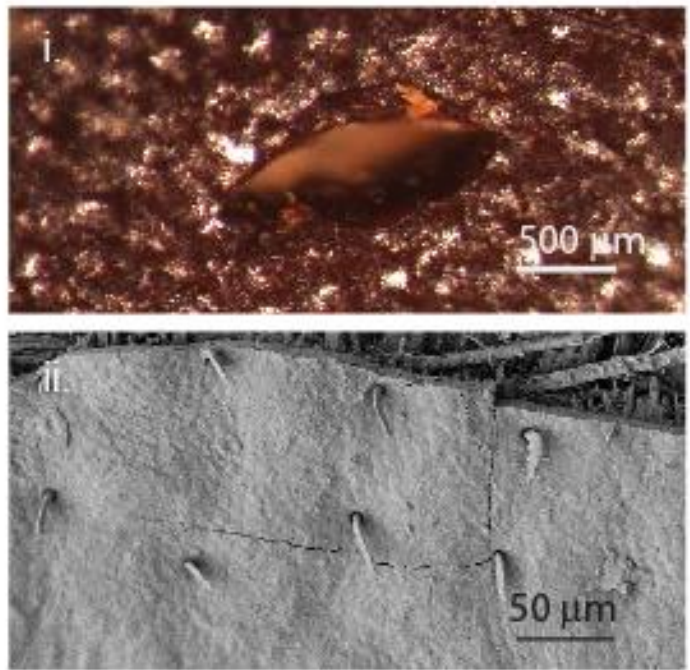

\section{D. i)Punctured hole ii) SEM image of fractured top layer}

Figure 8. A) Schematic showing the experimental set up used for puncture tests. B) Force curves from the puncture tests, showing the puncture force (highlighted by *). C) Showing the snapshots from the video during puncturing. D) i. Beetle top surface with the punctured hole showing the brittle nature of the top layer, ii. Brittle nature of the top layer is also observed from a fractured sample. 


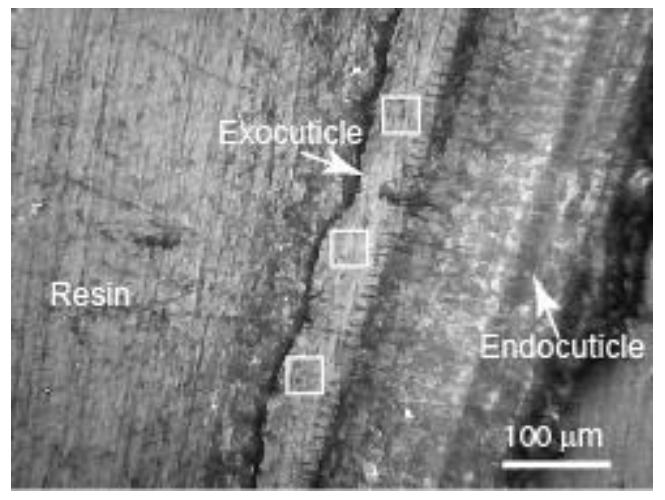

Figure 9. Optical image of the polished surface with the cuticle cross-section showing the selected locations of the nanoindentation on the external exocuticle.

\section{Conclusions}

In this study, we explored the mechanical properties of the stag beetle elytra which were not addressed in earlier studies that would aid in performing different functions. Our experimental results showed that the elytra deform using a unique snap-through mechanism that enables energy absorption during impacts and also retain their shape after impacts. Our finite element simulations showed that the elytra can undergo deformation using snap-through mechanism even if it has lower mechanical properties as compared to the properties reported in our dehydrated specimens. We also showed that the elytra support additional functions, such as puncture resistance and wear resistance. Such studies will help in designing of future bio-inspired materials and structures which could be not only shape retaining but also wear and puncture resistant. Future studies aimed at understanding the elytra layer-layer interface mechanisms under large deformation would aid in design of special application composites.

Funding: NMP is supported by the European Commission under the Graphene Flagship Core 2 No. 785219 (WP14 "Composites") and FET Proactive "Neurofibres" Grant No. 732344 as well as by the Italian Ministry of Education, University and Research (MIUR), under the "Departments of Excellence" grant L. 232/2016. R.G. is supported by Bonfiglioli Riduttori SpA.

Acknowledgments: The authors thank Nicola Angeli (MUSE, Trento, Italy) for the help with SEM imaging and Michele Menegon (MUSE, Trento, Italy) for the help in acquiring the samples.

Conflicts of Interest: The authors declare no conflict of interest. 


\section{References}

1. Hunt $\mathrm{T}$ et al. 2007 A comprehensive phylogeny of beetles reveals the evolutionary origins of a superradiation. Science 318, 1913-1916. (doi:10.1126/science.1146954)

2. Vincent JF V, Wegst UGK. 2004 Design and mechanical properties of insect cuticle. Arthropod Struct. Dev. 33, 187-99. (doi:10.1016/j.asd.2004.05.006)

3. Kamp T Van De, Greven H. 2010 On the architecture of beetle elytra. Entomol. heute 22, 191-204.

4. Lomakin J, Huber P a, Eichler C, Arakane Y, Kramer KJ, Beeman RW, Kanost MR, Gehrke SH. 2011 Mechanical properties of the beetle elytron, a biological composite material. Biomacromolecules 12, 321-35. (doi:10.1021/bm1009156)

5. Lomakin J, Arakane Y, Kramer KJ, Beeman RW, Kanost MR, Gehrke SH. 2010 Mechanical properties of elytra from Tribolium castaneum wild-type and body color mutant strains. J. Insect Physiol. 56, $1901-6$. (doi:10.1016/j.jinsphys.2010.08.012)

6. Hu N, Burgueño R. 2015 Buckling-induced smart applications: recent advances and trends. Smart Mater. Struct. 24, 063001. (doi:10.1088/0964-1726/24/6/063001)

7. Overvelde JTB, Kloek T, D'haen JJ a, Bertoldi K. 2015 Amplifying the response of soft actuators by harnessing snap-through instabilities. Proc. Natl. Acad. Sci. U. S. A. 112, 10863-8. (doi:10.1073/pnas.1504947112)

8. Brinkmeyer A, Santer M, Pirrera A, Weaver PM. 2012 Pseudo-bistable self-actuated domes for morphing applications. Int. J. Solids Struct. 49, 1077-1087. (doi:10.1016/j.ijsolstr.2012.01.007)

9. Hufenbach W, Gude M, Czulak A. 2006 Actor-initiated snap-through of unsymmetric composites with multiple deformation states. J. Mater. Process. Technol. 175, 225-230. (doi:10.1016/j.jmatprotec.2005.04.025)

10. McKittrick J, Chen PY, Tombolato L, Novitskaya EE, Trim MW, Hirata GA, Olevsky EA, Horstemeyer MF, Meyers MA. 2010 Energy absorbent natural materials and bioinspired design strategies: A review. Mater. Sci. Eng. C 30, 331-342. (doi:10.1016/j.msec.2010.01.011)

11. Correa DM et al. 2015 Mechanical design of negative stiffness honeycomb materials. Integr. Mater. Manuf. Innov. 4, 10. (doi:10.1186/s40192-015-0038-8)

12. Pini V, Ruz JJ, Kosaka PM, Malvar O, Calleja M, Tamayo J. 2016 How two-dimensional bending can extraordinarily stiffen thin sheets. Sci. Rep. 6, 1-6. (doi:10.1038/srep29627)

13. Forterre Y, Skotheim JM, Dumais J, Mahadevan L. 2005 How the Venus flytrap snaps. Nature 433, 421-426. (doi:10.1038/nature03072.1.)

14. Chen IH, Kiang JH, Correa V, Lopez MI, Chen PY, McKittrick J, Meyers MA. 2011 Armadillo armor: Mechanical testing and micro-structural evaluation. J. Mech. Behav. Biomed. Mater. 4, $713-722$. (doi:10.1016/j.jmbbm.2010.12.013)

15. Krauss S, Monsonego-Ornan E, Zelzer E, Fratzl P, Shahar R. 2009 Mechanical function of a complex threedimensional suture joining the bony elements in the shell of the red-eared slider turtle. Adv. Mater. 21, 407-412. (doi:10.1002/adma.200801256)

16. Amini S, Miserez A. 2013 Wear and abrasion resistance selection maps of biological materials. Acta Biomater. (doi:10.1016/j.actbio.2013.04.042)

17. Müller M, Olek M, Giersig M, Schmitz H. 2008 Micromechanical properties of consecutive layers in specialized insect cuticle: the gula of Pachnoda marginata (Coleoptera, Scarabaeidae) and the infrared sensilla of Melanophila acuminata (Coleoptera, Buprestidae). J. Exp. Biol. 211, 2576-83. (doi:10.1242/jeb.020164)

18. Ha NS, Le VT, Goo NS. 2018 Investigation of Punch Resistance of the Allomyrira dichtoloma Beetle Forewing. 15, 57-68.

19. Zhu D, Szewciw L, Vernerey F, Barthelat F. 2013 Puncture resistance of the scaled skin from striped bass: Collective mechanisms and inspiration for new flexible armor designs. J. Mech. Behav. Biomed. Mater. 24, 3040. (doi:10.1016/j.jmbbm.2013.04.011)

20. Clark AJ, Triblehorn JD. 2014 Mechanical properties of the cuticles of three cockroach species that differ in their wind-evoked escape behavior. PeerJ 2, e501. (doi:10.7717/peerj.501) 
21. Kundanati L, Signetti S, Gupta HS, Menegon M, Pugno NM. 2018 Multilayer stag beetle elytra perform better under external loading via non- symmetric bending properties. R. Soc. Interface

22. H. Fabritius, C. Sachs, D. Raabe, S. Nikolov MF, and J. Neugebauer. 2011 Chitin. p. 182. (doi:10.1007/97890-481-9684-5)

23. Riks E. 1979 An incremental approach to the solution of snapping and buckling problems. Int. J. Solids Struct. 15, 529-551. (doi:10.1016/0020-7683(79)90081-7)

24. Dai Z, Yang Z. 2010 Macro- / Micro-Structures of Elytra , Mechanical Properties of the Biomaterial and the Coupling Strength Between Elytra in Beetles. J. Bionic Eng. 7, 6-12. (doi:10.1016/S1672-6529(09)60187-6)

25. Gorb SN. 1998 Frictional Surfaces of the Elytra-to-Body Arresting Mechanism in Tenebrionid beetles (Coleoptera: Tenebrionidae) : Design of co-opted fields of microtrichia and cutilcle ultrastructure. Int. J. Insect Morphol. Embryol. 27.

26. Schultz MR, Hyer MW. 2003 Snap-through of unsymmetric cross-ply laminates using piezoceramic actuators. J. Intell. Mater. Syst. Struct. 14, 795-814. (doi:10.1177/104538903039261)

27. Brampton CJ, Betts DN, Bowen CR, Kim HA. 2013 Sensitivity of bistable laminates to uncertainties in material properties, geometry and environmental conditions. Compos. Struct. 102, $276-286$. (doi:10.1016/j.compstruct.2013.03.005)

28. Dano ML, Hyer MW. 2003 SMA-induced snap-through of unsymmetric fiber-reinforced composite laminates. Int. J. Solids Struct. 40, 5949-5972. (doi:10.1016/S0020-7683(03)00374-3)

29. Arena G, Groh RMJ, Brinkmeyer A, Theunissen R, Weaver PM, Pirrera A. 2017 Adaptive compliant structures for flow regulation. Proc. R. Soc. A Math. Phys. Eng. Sci. 473, 20170334. (doi:10.1098/rspa.2017.0334)

30. Bellini PX. 1972 The concept of Snap-buckling illustrated by a simple model. Int. J. Non. Linear. Mech. 7, 643-650.

31. Bazzucchi F, Manuello A, Carpinteri A. 2017 Interaction between snap-through and Eulerian instability in shallow structures. Int. J. Non. Linear. Mech. 88, 11-20. (doi:10.1016/j.ijnonlinmec.2016.10.006)

32. Sumirin, Nuroji, Besari S. 2015 International Journal of Science and Engineering ( IJSE ) Snap-Through Buckling Problem of Spherical Shell Structure. Int. J. Sci. Eng. 8, 54-59. (doi:10.12777/ijse.8.1.54-59)

33. Di Landro L, Sala G, Olivieri D. 2002 Deformation mechanisms and energy absorption of polystyrene foams for protective helmets. Polym. Test. 21, 217-228. (doi:10.1016/S0142-9418(01)00073-3)

34. Broomell CC, Zok FW, Waite JH. 2008 Role of transition metals in sclerotization of biological tissue. Acta Biomater. 4, 2045-2051. (doi:10.1016/j.actbio.2008.06.017)

35. Chen P-Y, Lin AY-M, McKittrick J, Meyers MA. 2008 Structure and mechanical properties of crab exoskeletons. Acta Biomater. 4, 587-96. (doi:10.1016/j.actbio.2007.12.010)

36. Klocke D, Schmitz H. 2011 Water as a major modulator of the mechanical properties of insect cuticle. Acta Biomater. 7, 2935-42. (doi:10.1016/j.actbio.2011.04.004)

37. Politi Y, Priewasser M, Pippel E, Zaslansky P, Hartmann J, Siegel S, Li C, Barth FG, Fratzl P. 2012 A spider's fang: How to design an injection needle using chitin-based composite material. Adv. Funct. Mater. 22, 25192528. (doi:10.1002/adfm.201200063)

38. Lichtenegger HC, Schöberl T, Bartl MH, Waite H, Stucky GD. 2002 High abrasion resistance with sparse mineralization: copper biomineral in worm jaws. Science 298, 389-92. (doi:10.1126/science.1075433)

39. Meng J, Zhang P, Wang S. 2015 Recent progress of abrasion-resistant materials: learning from nature. Chem. Soc. Rev. 45, 237-251. (doi:10.1039/C5CS00459D) 